\title{
Time to Respond
}

\section{Artifact Free Transient Near-Field Nanoscopy}

Authors: A. Sternbach, J. Hinton, T. Slusar, A. Swinton McLeod, M. Liu, A. Frenzel, M. Wagner, R. Iraheta, F. Keilmann, A. Leitenstorfer, M. Fogler, H.-T. Kim, R.

Averitt, D. N. Basov

arXiv:1706.08478 (2017)

\section{Photonic crystals for nano-light in moir graphene superlattices}

Authors: S. S. Sunku, G. X. Ni, B. Y. Jiang, H. Yoo, A. Sternbach, A. S. McLeod, T. Stauber, L. Xiong, T. Taniguchi, K. Watanabe, P. Kim, M. M. Fogler, D. N. Basov Science 362, 1153 (2018)

\section{Recommended with a Commentary by Dirk van der Marel, University of Geneva}

In a recent paper Sternbach and collaborators describe a novel major step in optical spectroscopy: time and space resolved optical spectroscopy combining ultra-fast time resolution with nano scale space resolution [1]. Time resolved optical spectroscopy [2, 3] and near-field optics $[4,5,6]$ have evolved independently from each other over the past decades. Sternbach et al. show that intelligent use of time-domain sampling allows to realize artifact-free time-resolved near-field spectra and images for infrared light. Their new approach brings also closer the realization of an old experimentalist's dream: To follow in real time the propagation and diffusion of a quasi-particle in a solid.

A recurrent quantity in our physics classes are the socalled Green functions, named after George Green, brillant self-taught mathematician and physicist of the early 19th century (see Ref. [8] for a description of his life and work). He introduced these functions in his Essay on the Applications of Mathematical Analysis to the Theories of Electricity and Magnetism [9] as a mathematical tool to solve electrostatic problems in bounded regions. The concept was later generalized to many other situations. In condensed matter physics and in elementary particle physics the Green function is alternatively coined Feynman propagator. The concept is illustrated in Fig. 1: Imagine that we inject an elementary particle (electron, hole, photon [10]) or a composite one (e.g. an electron-

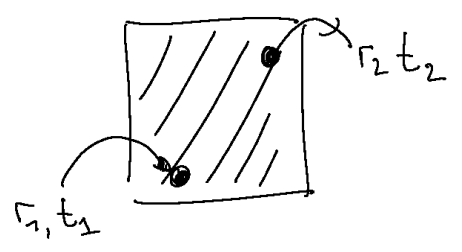
hole pair) at coordinate $r_{1}$ and time $t_{1}$. We let the resulting system evolve, and we annihilate 
an identical elementary or composite particle at time $t_{2}$ and coordinate $r_{2}$. The Green function $G\left(r_{1}, r_{2} ; t_{1}, t_{2}\right)$ corresponds to the amplitude and the phase of such a process.

The usefulness is manifold: When applied to holes, the imaginary part of its' space-time Fourier transform corresponds exactly to the spectral function that experimentalists measure using angle-resolved photo-emission spectroscopy. When applied to electron-hole pairs one obtains the (spin- or charge) susceptibility, and so on and so forth. The validity of the concept does not depend on whether the material is ordered, nor on the shape and the size of the sample, nor on the question whether the system is strongly or weakly interacting; it even applies when none of the excitations have any electron- or hole character. The way the Green function propagates does however depend on all of these factors, and as such it constitutes a powerful - and well defined- probe of these different influences. At the same time it also constitutes a quantity which is very close to the "work floor" of engineers who want to inject electrons or holes through an electrical contact, and extract them at some other location after manipulating them with a gate voltage or whichever knob they need to operate their micro-electronic devices.

Having said that, the principle of linear superposition is one of the basic tenets of the Green function formalism. Correspondingly the realms of linear response represent the safe haven of their applicability. This limitation may become important if, for example, the addition of a single electron would bring the system far out of equilibrium. While for experiments such as ARPES this is usually not considered an issue, in non-linear optics it is an issue by definition. We will return to this point at the end of this Commentary, but first we explore a bit further the linear-response. Here comes the snag: We usually instruct our students to "imagine" the experiment of Fig. 1, and we usually continue to tell them that in practice such an experiment is not possible. We go on to state that fortunately it is possible to measure the Fourier-transformed quantity as a function of frequency and momentum, and that such a quantity is in fact just as nice, or perhaps even better. In this sence Fig. 1 constitutes a "Gedanken experiment". Or at least, so it did until recently because Sternbach's ploy comes close to the ideal of measuring directly the propagation as a function of time. In their case the experiment, sketched in Fig. 2, involves -instead of an electron or a holea neutral excitation, i.e. an electron-hole pair in its' simplest incarnation. Their scheme consists of creating the excitation at coordinate $r_{1}$ and detecting it at a later time at the same location, $r_{2}=r_{1}$. Indeed, the most common modus operandi of near field (infrared) optics is to exploit the confining properties of the near-field tip to narrow down the field of vision to nanometer size, and scan the tip over the sample to create an image. This has allowed to map among other things phase-separation in metal-insulator transitions [5], to detect surface plasmons on graphene including -most recently- plasmon interference patterns at $\mathrm{AB} / \mathrm{BA}$ domain walls of bilayer graphene [6] and even create photonic crystals based on this effect [7]. However, it is possible to generalize this scheme in such a way as to have $r_{2} \neq r_{1}$. Given that momentum dependent information is encoded in tip- and sample geometry, it is possible with a stretch of imagination to decode this information, provided one has a way of modulating the geometrical parameters of the experiment. Hence, it is time to say farewell to the label "Gedanken experiment" when introducing Green functions, since this type of experiment has -as of now- landed very much on the real axis of laboratory practice.

The authors of Ref. [1] concentrate their discussion on heterodyne detection schemes 


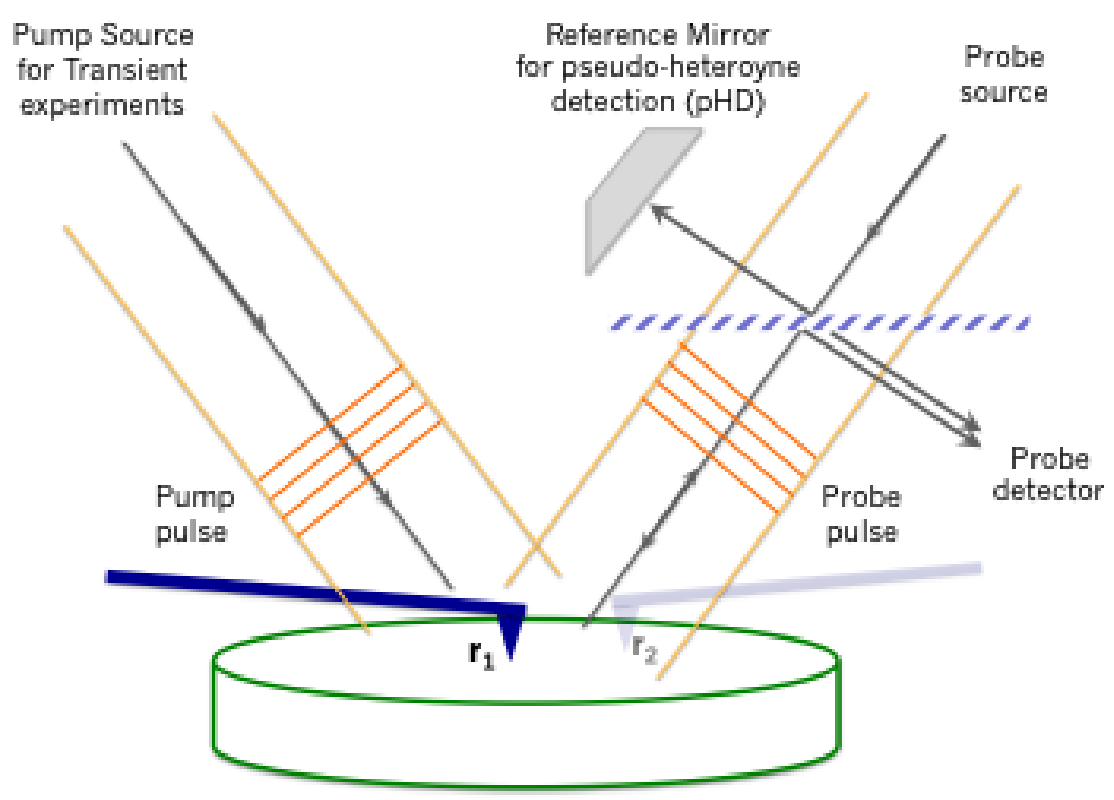

Figure 2: Sketch of the experimental rig of Sternbach et al.. Current experimental state of the art provides a time resolution $\Delta t \geq 4$ fs and space resolution $\Delta x \geq 10 \mathrm{~nm}$. The additional AFM tip at the righthand side (shaded, not in Sternbach's setup) could serve as a tool to address $r_{2} \neq r_{1}$.

allowing them to separate near-field images/spectra from (unwanted) additive background contributions. The probe signals in this case are supposed to be sufficiently weak to remain in the regime of linear response of the material. However, given the composition and expertise of the team of authors of this paper one's thoughts naturally wander off to the myriads of opportunities provided by non-linear optics. The authors do in fact discuss pump-probe experiments using ultra short light pulses. While the probe part of the experiment may still be a linear response, the material is driven out of equilibrium by the pump pulse. It requires a small stretch of imagination to anticipate that this field will continue to evolve, including higher harmonic generation experiments in a near-field optical rig.

\section{References}

[1] A. Sternbach, J. Hinton, T. Slusar, A. Swinton McLeod, M. Liu, A. Frenzel, M. Wagner, R. Iraheta, F. Keilmann, A. Leitenstorfer, M. Fogler, H.-T. Kim, R. Averitt, D. N. Basov, Artifact Free Transient Near-Field Nanoscopy, arXiv:1706.08478 (2017).

[2] C. K. Sun, F. Vallee, L. Acioli, E. P. Ippen, and J. G. Fujimoto, Femtosecond investigation of electron thermalization in gold, Phys. Rev. B 48, 12365 (1993).

[3] R. D. Averitt, and A. J. Taylor, Ultrafast optical and far-infrared quasiparticle dynamics in correlated electron materials, J. Phys. Condens. Matter 14, R1357 (2002). 
[4] F. Keilmann, R. Hillenbrand, Near-field microscopy by elastic light scattering from a tip, Philos. Trans. R. Soc. London Ser. A 362, 787 (2004).

[5] M. M. Qazilbash, M. Brehm, B.-G. Chae, P.-C. Ho, G. O. Andreev, B.-J. Kim, S. J. Yun, A. V. Balatsky, M. B. Maple, F. Keilmann, H.-T. Kim, and D. N. Basov, Mott Transition in $\mathrm{VO}_{2}$ Revealed by Infrared Spectroscopy and Nano-Imaging, Science 318, 1750 (2007).

[6] B.-Y. Jiang, G.-X. Ni, Z. Addison, J. K. Shi, X. Liu, S. Yang, F. Zhao, P. Kim, E. J. Mele, D. N. Basov, and M. M. Fogler, Plasmon Reflections by Topological Electronic Boundaries in Bilayer Graphene, Nano Letters 17, 7080 (2017).

[7] S. S. Sunku, G. X. Ni, B. Y. Jiang, H. Yoo, A. Sternbach, A. S. McLeod, T. Stauber, L. Xiong, T. Taniguchi, K. Watanabe, P. Kim, M. M. Fogler, D. N. Basov, Photonic crystals for nano-light in moir graphene superlattices, Science 362, 1153 (2018).

[8] L. Challis and F. Sheard, The Green of Green Functions, Physics Today 56, 44 (2003).

[9] G. Green, An Essay on the Applications of Mathematical Analysis to the Theories of Electricity and Magnetism, printed for the author by T. Wheelhouse, Nottingham (1828).

[10] J. D. Jackson, Classical Electrodynamics, J. Wiley \& sons, New York (1962, 1975, 1998).

[11] D. van der Marel and T. Giamarchi, Advanced Solid State Physics II: electronic structure, http://www.dirkvandermarel.ch/resources/ASSP2018.pdf. 